so knapp sind, daß man über den Mut der Autoren staunen kann. Bei manchen Artikeln wäre es besser gewesen, die persönliche Auffassung des Autors nicht so sehr in den Vordergrund zu schieben und auch die Auffassung anderer Autoren, die sich ebenfalls Jahrzehnte hindurch mit den Problemen beschäftigten, zu berücksichtigen. In diesen Fällen wäre mehr Objektivität und mehr Toleranz sympathischer gewesen.

Der Inhalt des Bandes sei kurz angedeutet. Der Ref. hatte gerne zum einen oder anderen Beitrag seine Meinung gesagt, doch würde das den Rahmen eines übersichtlichen Referates sprengen.

W. SeIFrIz $†$ liefert eine Zusammenfassung uber die Energieprobleme. Die Oberflächenspannung, der Diffusionsdruck, die Potentiale und bioelektrischen Ströme sowie die Energetik des Protoplasmas werden in ausgezeichneter Weise abgehandelt. C. R. Stocking berichtet uber die Probleme der Wasserfuihrung der Zelle. B. S. Meyer bringt ein Kapitel über den Wand- und Turgordruck, wobei auch die Tagesperiodizitat berücksichtigt wird. Der osmotische Druck und der osmotische Wert werden von C. R. StockIng sehr klar abgehandelt. E. Stadelmann gibt einen Abriß uber die Plasmolyse und Deplasmolyse. Die Literatur wird gut ausgewertet, und alle wichtigen Phänomene werden klar und übersichtlich besprochen.

H. J. Bogen gibt eine Einführung in das Problem der Permeabilität und der nichtosmotischen Stoffaufnahme und -abgabe. Hier erfährt man sehr konzentriert die persönliche Auffassung des Autors. Es ist interessant, in diesem Artikel zu erfahren, daß der Begriff Intrabilität dem Autor deshalb nicht wichtig erscheint, we1l man den Vorgang zwar wohl beobachten, aber nicht messen kann. Wenn man weiß, wieviel im kolloidchemischen Zeitalter der Zellphysiologie umsonst gemessen wurde und wie viele dieser Messungen falsch waren, so ist eine solche Art der Bewertung zellphysiologischer Phänomene etwas bedenklich. Bei der Frage, ob osmotische oder anosmotische Prozesse vorherrschen, rät der Ref. von allzu extremen Deutungen ab. Es sind zweifellos beide Prozesse wichtig, beide Vorgänge beherrschen je nach der physiologischen Situation der Zelle das Feld.

D. C. Spanner behandelt die mathematischen Grundlagen der Diffusionsvorgänge und E. STADELMANN die mathematische Analyse der experimentellen Ergebnisse der Permeabilitätsforschung. Diese Kapitel sind dankenswerte und höchst nützliche Beiträge. Die Methode der Messung des Stoffaustausches faßt R. Collander kurz zusammen. Vom gleichen Autor wird die schwierige Frage des Ortes des Penetrationswiderstandes besprochen. Collander spricht den Plasmagrenzschichten die Hauptrolle zu, womit z.B. H.S. Troschin sicherlich nicht einverstanden sein wird. Die Aufnabme der Anelektrolyten wird von H. J. Bogen unter sehr breiter Heranziehung der Literatur mit anerkennenswerter Objektivität besprochen. Das Kapitel uber die Vitalfärbung wird von $H$. DRAWERT bearbertet. Es ist klar gegliedert, und man erfährt daraus alles, was wichtig ist. P. J. KRAMER behandeit die Salz- und Wasseraufnahme durch die Zelle. L. BraUner informiert sehr gründlich über die Permeabilitätseigenschaften der Zellwand. Die Beziehungen zwischen der Permeabilität und der Atmung werden von P. J. Kramer besprochen. V. Wartiovaara und L. Brauner behandeln das Licht als beeinflussenden Faktor des Stoffaustausches. In klassischer Kürze wird von E. Epstern das Kapitel Stoffaufnahme und Ionenmilieu einschließlich des $\mathrm{p}_{\mathrm{H}}$-Wertes des Mediums behandelt (8 Seiten). H. DrawerT berichtet über den Zusam- menhang zwischen innerem $\mathrm{p}_{\mathbf{H}^{-}}$Wert der Zelle und Stoffaufnahme.

Darauf folgt ein Kapitel von E. BúnNing uber die Beeinflussung der Stoffaufnahme durch den physiologischen $\mathrm{Zu}$ stand. H. J. Bogen berichtet in einem Kapitel iber die Objekttypen der Permeabilitat sowie in einem weiteren Kapitel über die spezifischen Permeabilitätsreihen, wobei die Waage der Objektivitat gegenüber anderen Forschern nicht ganz im Gleichgewicht ist, ferner über die Theorien der Permeabilität. Der Mechanismus der Absorption wird von R. N. RoBertson behandelt. Darauf folgt ein Kapitel von R. J. HeLder uber die Salzdrüsenausscheidung, ein Artikel, den ich als ausgezeichnet empfunden habe.

Ein großer Gewinn fur das Handbuch ist der Artikel von F. Duspiva, der die Bedeutung der Enzyme im Zellstoffwechsel behandelt. Daran schließt sich ein Kapitel uber die Biochemie des Zellkernes an, welches aus der Feder von G. F. BaHR stammt.

ADÈLE MILIFRD behandelt vom rein biochemischen Standpunkt aus die Mitochondrien und die Mrikrosomen. Der Ref. muß aber hervorheben, daß hier der Ausdruck Mikrosomen nicht im Sinne der botanischen Cytologie gebraucht ist. Es handelt sich vielmehr um sublichtmıkroskopische Partıkeln, welche nach Homogenisation aus dem Plasma isoliert werden können. Nun wird in je einem weiteren Kapitel uber die $\mathrm{Hy}$ dratation von Zellbestandteilen durch J.LEviTr, uber die Viskosität des Plasmas durch M. G. STÅLfELT berichtet.

Gleich darauf folgt unter dem anspruchsvollen Titel ,Koordination der Reaktionssysteme" ein Kapitel von H. J. BoGEN, welches in diesem Zusammenhang durch seine Kurze und durch das Fehlen vieler in der Literatur auffindbarer Zusammenhänge nicht ganz glücklich gelungen ist.

J. Levitr behandelt in Auswahl die Probleme der Hitzeund Kälteresistenz der Zelle. O. STocker gibt einen guten Emblick in das Kapitel Wassermangel und Zellaktivität. W. Sumonis verdanken wir ein sehr umfangreiches und sehr gewissenhaft geschriebenes Kapitel über die Wirkung von Licht und Strahlung auf die Zelle. Anschließend gibt H. FISCHER einen guten Überblick über die Ionenwirkung. Die elektrophysiologischen Phänomene sind von K. UMRATH in vorbildlich klarer Weise abgehandelt. Narkose und Narkotica werden von K. PAECH $†$ bearbeitet. Leider ist der Autor $z u$ fruh verstorben, so daß V. Wartiovaara und R. CollanDER es ubernommen haben, das überlassene Manuskriptfragment glücklich $\mathrm{zu}$ vollenden. H. P. CuRRIER berichtet uber die Guftwirkung verschiedener toxischer Substanzen sowie uber andere Zellschädigungen. Das Kapitel ist sehr interessant und originell geschrieben und bringt viele wertvolle Literaturhinweise.

Ein schönes Kapitel über die Resistenz gegen Infektionen und über die Frage der Immunitat verdanken wir H. KERN. Ủber das Vorkommen und die Arten des Aktivitatswechsels, uber regulierende Faktoren des Aktivitätswechsels sowie über endogene Aktivitatsrhythmen gibt E. BüNNING ein gründlich bearbeitetes und mit großem Genuß zu lesendes Kapitel.

Abgeschlossen wird das Handbuch durch einen Abschnitt über Altern und Tod von K. PAECH $t$ und F. EBERHARD.

Das Buch ist in seiner Ausstattung hervorragend. Das Sachverzeichnis ist ausgezeichnet und verläßlich, und wir kömnen wirklich glucklich sein, daß eine derartige Zusammenfassung gewagt und praktisch durchgeführt wurde.

S. STrugger (Münster)

\title{
Berichtigung und Ergänzung
}

zu dem Aufsatz ,Wesen und Anwendung der Beugungsmikroskopie im Gesamtbereich von den sichtbaren bis zu den $\gamma$ Strahlen“ von R. Hosemann, Naturwiss. 46, 542 (1959): 1. In Fig. 2 muß an dem nach unten weisenden Vektor $\vec{b} \lambda$ stehen, nicht $\vec{b}$. -2 . Ersetze in Fig $5 \mathrm{~b} \mathrm{Cl} \mathrm{Cl}^{+}$durch $\mathrm{Cl}^{-}$und $\mathrm{Na}^{-}$durch $\mathrm{Na}^{+}$. - 3. Ersetze in der Legende zu Fig. $5 \mathrm{~d}$ das Zeichen. (-...-) für , experimentell" durch (- $(-$ Die gestrıchelten Kurven stammen aus einer Gauß-Analyse (Näheres s. [19c]). 4. Vertausche auf S. 547, linke Spalte, vorletzte Zeile die Zeichen $\mathrm{Na}^{+}$und $\mathrm{Cl}^{-}$miteinander. - 5. Herr M. BaLARIN (Zentralinstitut für Kernphysik, Rossendorf bei Dresden) machte mich in freundlicher Weise darauf aufmerksam, daß der in Fig. $5 \mathrm{~d}$ fur $\mathrm{Cl}^{-}$ und $\mathrm{Na}^{+}$experimentell gefundene Deformationseffekt von M.I. Petrashen, A.V. Iwanowa und H. Wolf [Nachr. Leningr. Univ. No. 10.29 (1956)] mittels der Hartree-Fock-Methode theoretisch berechnet wurde. Sie fanden in Übereinstimmung mit dem experimentellen Befund der Fig. 5 d, daß ganz allgemein in Ionenkristallen die äußeren Elektronenschalen der Anıonen zusammengedrängt, diejenigen der Kationen aufgelockert werden R. HOSEMANN 